\title{
Overexpression of Batf induces an apoptotic defect and an associated lymphoproliferative disorder in mice
}

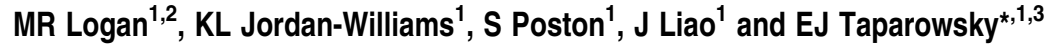

Activator protein-1 (AP-1) is a dimeric transcription factor composed of the Jun, Fos and Atf families of proteins. Batf is expressed in the immune system and participates in AP-1 dimers that modulate gene expression in response to a variety of stimuli. Transgenic $(\mathrm{Tg})$ mice overexpressing human BATF in T cells were generated using the human CD2 promoter (CD2-HA (hemagglutinin antigen) - BATF). By 1 year of age, over $90 \%$ of the mice developed a lymphoproliferative disorder (LPD). The enlarged lymph nodes characteristic of this LPD contain a polyclonal accumulation of T cells with a CD4 ${ }^{+}$bias, yet efforts to propagate these tumor cells in vitro demonstrate that they do not proliferate as well as wild-type $\mathrm{CD} 4^{+} \mathrm{T}_{\text {cells. Instead, the }}$ accumulation of these cells is likely due to an apoptotic defect as CD2-HA-BATF $\mathrm{Tg}$ T cells challenged by trophic factor withdrawal in vitro resist apoptosis and display a pro-survival pattern of Bcl-2 family protein expression. As elevated levels of Batf expression are a feature of lymphoid tumors in both humans and mice, these observations support the use of CD2-HA-BATF mice as a model for investigating the molecular details of apoptotic dysregulation in LPD.

Cell Death and Disease (2012) 3, e310; doi:10.1038/cddis.2012.49; published online 17 May 2012

Subject Category: Cancer Metabolism

Activator protein-1 (AP-1) is a dimeric transcription factor that is comprised of ubiquitous and tissue-specific basic leucine zipper proteins of the Jun, Fos, Maf and Atf families of proteins. ${ }^{1}$ In response to many stimuli, AP-1 component expression and DNA-binding activity are induced rapidly in cells and result in both the positive and negative regulation of gene expression. ${ }^{2}$ In lymphocytes, AP-1 activity increases following B-cell and T-cell receptor (TCR) engagement and participates in a transcription factor network that regulates the expression of genes associated with lymphocyte activation and differentiation. ${ }^{2,3}$ The AP-1 protein Batf is expressed in both $T$ and $B$ cells and although there is compelling evidence to suggest that AP-1 dimers in which Batf participates are transcriptionally inactive, ${ }^{4,5}$ the recent identification of Batf genetic targets in vivo indicates that Batf also may function as a positive regulator of gene expression. ${ }^{6,7}$ The role for Batf in the development and differentiation of lymphocytes has been investigated by several groups using T-cell-specific Batf transgenic $(\mathrm{Tg})^{8,9}$ and systemic Batf knock-out mice. $6,7,10,11$ Results show that increased expression of Batf in $\mathrm{T}$ cells impacts signaling important to the proper development of thymic NKT cells ${ }^{8,9}$ and that the loss of Batf has a dramatic impact on the differentiation of $\mathrm{CD}^{+}{ }^{+}, \mathrm{IL}-17$-producing T-helper cells (Th17) and T-follicular T-helper cells (Tfh) in the periphery. ${ }^{7,10}$ Additionally, Batf null animals possess an intrinsic B-cell defect that cooperates with the Th17 and Tfh-cell deficiencies to inhibit antibody responses to both T-cell-dependent and T-cell-independent antigens. ${ }^{6,10}$ In conjunction with these studies, an analysis of gene expression changes in Batf null lymphocytes identified several genes in $T$ cells and B cells whose expression is correlated with the binding of Batf to specific gene regulatory regions. ${ }^{6,7}$ Precisely how the Batf protein directs the transcription of these target genes, however, is not known.

Previous studies on the function of Batf in vivo have focused primarily on phenotypes that are apparent in the mouse immune system by 4 months of age. In this study, we have utilized a line of $\mathrm{Tg}$ mice in which the human BATF protein is expressed constitutively in both thymic and peripheral $T$ cells to test the impact of long-term, chronic BATF overexpression (and AP-1 mis-regulation) on T-cell maturation and homeostasis. Remarkably, we found that by 12 months of age, the vast majority of these animals develop lymphoid tumors consisting of a polyclonal population of T cells. Our studies demonstrate that this lymphoproliferative disorder (LPD) is not associated with an increase in the proliferative capacity of the $T$ cells, but rather with an impaired response to an apoptotic stimulus. Given the observation that increased

\footnotetext{
${ }^{1}$ Department of Biological Sciences, College of Science, Purdue University, West Lafayette, IN, USA; ${ }^{2}$ Department of Comparative Pathobiology, School of Veterinary Medicine, Purdue University, West Lafayette, IN, USA and ${ }^{3}$ Purdue University Center for Cancer Research, Purdue University, West Lafayette, IN, USA ${ }^{*}$ Corresponding author: EJ Taparowsky, Department of Biological Sciences, College of Science, Purdue University, 201 South University Street, West Lafayette, IN 47907-2064, USA. Tel: 765494 7978; Fax: 765496 2536; E-mail: taparows@ purdue.edu

Keywords: Batf; AP-1; lymphoproliferative disorder; ACAD; transgenic mice

Abbreviations: ACAD, activated cell autonomous death; AICD, activation-induced cell death; ALPS, autoimmune lymphoproliferative syndrome; ANA, anti-nuclear antibodies; AP-1, activator protein-1; BrdU, bromodeoxyuridine; EAE, experimental autoimmune encephalolmyelitis; ELISA, enzyme-linked immunosorbent assay; EMSA, electrophoretic mobility shift assays; H\&E, hematoxylin and eosin; HA, hemagglutinin antigen; IHC, immunohistochemistry; LPD, lymphoproliferative disorder; MRI, magnetic resonance imaging; PCR, polymerase chain reaction; ROS, reactive oxygen species; RT-qPCR, quantitative real-time polymerase chain reaction; TCR, T-cell receptor; Tfh, follicular T-helper cells; Tg, transgenic; Th17, IL-17-producing T-helper cells; TUNEL, terminal deoxynucleotidyl transferase dUTP nick end-labeling; WT, nontransgenic control
}

Received 28.2.12; revised 02.4.12; accepted 02.4.12; Edited by H-U Simon 
BATF expression is a feature of secondary neoplasias in human Hodgkin's lymphoma ${ }^{12}$ and of MuMLV-induced T-cell lymphoma in mice ${ }^{13}$ the BATF Tg mice described herein represent a useful model for investigating the role of AP-1 mis-regulation in LPD.

\section{Results}

Tg mice expressing hemagglutinin antigen (HA) -epitope tagged human BATF protein in T cells (CD2-HA-BATF) have been described previously in conjunction with a study that demonstrated a direct role for BATF in regulating thymocyte proliferation in vitro. ${ }^{14}$ For the current study, it was important to establish that the CD2-HA-BATF transgene continues to be expressed in peripheral T cells and that TCR signaling in $\mathrm{Tg} \mathrm{T}$ cells remains intact and properly induced by AP-1 complexes containing BATF. Splenocytes from $\mathrm{Tg}$, non- $\mathrm{Tg}$ (WT) littermates and Batf null mice were stimulated in vitro with anti-CD3 and anti-CD28 antibodies. Protein extracts were prepared and immunblotted using anti-BATF antiserum. Results show a robust overexpression of BATF protein in the Tg cells (Figure 1a). Nuclear extracts were prepared from resting and stimulated WT and Tg splenocytes and used for electrophoretic mobility shift assays (EMSA) to detect the presence of protein complexes competent for binding consensus AP-1 DNA (Figure 1b). In the absence of stimulation, the $\mathrm{Tg}$ nuclear extract showed increased AP-1 activity resulting from the formation of DNA-binding heterodimers containing human BATF and the Jun proteins expressed in resting $T$ cells. Following stimulation, the endogenous AP-1 factors, including Jun, Fos and Batf, are upregulated in both WT and Tg cells and the DNA-binding activity in these extracts increases accordingly. This DNAbinding activity is identified as AP-1 by successful competition with a 100-fold excess of unlabeled AP-1, but not SP-1, DNA. The presence of BATF is demonstrated by the higher molecular weight complexes detected following the addition of polyclonal anti-BATF or monoclonal anti-HA antiserum to the reaction (Figure $1 \mathrm{~b}$, complexes 1 and 2 , respectively). These results validate use of the Tg, CD2-HA-BATF mouse model to study the long-term impact of BATF expression on peripheral T-cell function.

Initial characterization of the effects of CD2-HA-BATF expression on peripheral lymphoid tissues was performed using histology and revealed a significantly reduced area of white pulp in $\mathrm{Tg}$ spleens (Figure 1c). This reduction was quantified to reveal that the total follicle area in $\mathrm{Tg}$ spleens was roughly $75 \%$ of WT controls (Figure 1c). To profile the splenic lymphocyte populations in CD2-HA-BATF mice, single-cell suspensions from 8-12-week-old mice were analyzed by flow cytometry. Although the total cellularity of $\mathrm{Tg}$ spleens is reduced compared with WT littermate controls (Figure 1d, left panel), the percentage of T cells in the spleens of Tg mice is increased (Figure 1d, center panel). Furthermore, $\mathrm{Tg}$ T cells show a statistically significant bias toward $\mathrm{CD}^{+}$cells (Figure 1d, right panel). These findings are consistent with the reduced percentage of splenic $\mathrm{CD}^{+} \mathrm{T}$ cells noted previously in Batf null $\left(\right.$ Batf $\left.^{\lrcorner Z / \Delta Z}\right)$ mice. ${ }^{10}$

In order to determine how BATF overexpression influences T-cell homeostasis, CD2-HA-BATF Tg mice, along with their
WT littermates, were allowed to age for up to 14 months. A significant decrease in body weight was observed in male Tg mice, whereas the average weight of female $\mathrm{Tg}$ mice was not reduced significantly compared with WT controls (Figure 2a). Upon necropsy, $91.6 \%$ of aged Tg mice of both sexes exhibited gross peripheral lymphadenopathy in at least one lymph node (Figure $2 b$ ). The affected nodes presented as single or multiple discrete tumors. Mesenteric nodes were most commonly affected by lymphadenopathy; however, disease also was observed in other peripheral lymph nodes and spleen. In addition to the gross lesions, microscopic evidence of lymphoid infiltrate was detected in the liver and pancreas of all tumor-bearing CD2-HA-BATF mice. Only three WT mice showed signs of a similar gross and/or microscopic disease at necropsy.

To evaluate the kinetics of tumor formation in $C D 2-H A$ $B A T F$ mice, a longitudinal magnetic resonance imaging (MRI) study was conducted for 10 months during which time $11 \mathrm{Tg}$ and 10 WT littermate control mice were imaged monthly beginning at 4 months of age. The results confirmed that the tumor phenotype was an exclusive feature of older $\mathrm{Tg}$ animals, as the median age at earliest tumor detection by this noninvasive method was 10 months (Figure 2c).

Histological characterization of the tumor tissue from CD2-HA-BATF mice revealed a heterogeneous population of small and intermediate sized lymphocytes, which completely disrupted the follicular architecture of the lymph node (Figure 2d, upper panels). Immunohistochemistry (IHC) identified the predominant tumor-cell population as $\mathrm{CD}^{+}{ }^{+}$cells although low, but variable, numbers of CD79a ${ }^{+}$ $B$ cells were found throughout the affected nodes (Figure 2d, middle panels). Importantly, the Tg HA-BATF protein was detected in a high percentage of the tumor cells by IHC (Figure 2d, lower panels) and flow cytometry on individual tumor-cell suspensions detected anti-HA reactive BATF protein, on average, in $68 \%$ of the $\mathrm{CD}^{+} \mathrm{T}$ cells analyzed (Figure 2e).

To augment the IHC results, cell suspensions from several tumors were analyzed using flow cytometry to quantify the relative percentages of $\mathrm{B}$ versus $\mathrm{T}$ lymphocytes and to quantify any bias toward CD4 ${ }^{+}$or $\mathrm{CD}^{+}{ }^{+}$cells. Pooled singlecell suspensions from the lymph nodes of aged WT mice were used as controls. As shown in Figure 3a, the tumors are composed primarily of $\mathrm{T}$ cells (upper panels) and display a bias towards $\mathrm{CD}^{+}$cells (lower panels). Interestingly, the CD4 and CD8 co-staining reveals a significant level ( $\sim 12 \%)$ of an uncharacterized, double-negative $\left(\mathrm{CD} 4^{-} \mathrm{CD} 8^{-}\right)$ $\mathrm{TCR} \beta^{+}$cell type in these tumors. The presence of these cells, together with both $\mathrm{CD} 4^{+}$and $\mathrm{CD} 8^{+}$single-positive $\mathrm{T}$ cells, indicates that these tumors do not arise from a single $\mathrm{T}$-cell clone as would be the case in T-cell lymphoma. To verify the polyclonal origin of the T cells in these masses, a PCRbased approach ${ }^{15}$ was used to detect rearrangements within the TCR $\beta$ locus (Figure $3 b$ ). Three primers sets spanning $\operatorname{TCR} \beta$ diversity (D) and junction (J) regions 1 and 2 were used to amplify fragments of the locus from tumor DNA. Controls included DNA representing the broad $\operatorname{TCR} \beta$ diversity of thymocyte DNA, the clonal TCR $\beta$ rearrangement from a mouse iNKT cell line and the nonrecombined, germ line TCR $\beta$ locus in mouse ES cell DNA (Figure $3 b$ ). The genomic DNA 
a

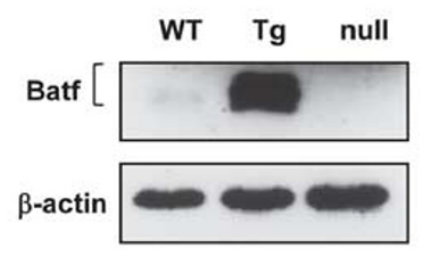

b

No Stim.

$\alpha-\operatorname{CD} 3 \varepsilon+\alpha-\operatorname{CD} 28$

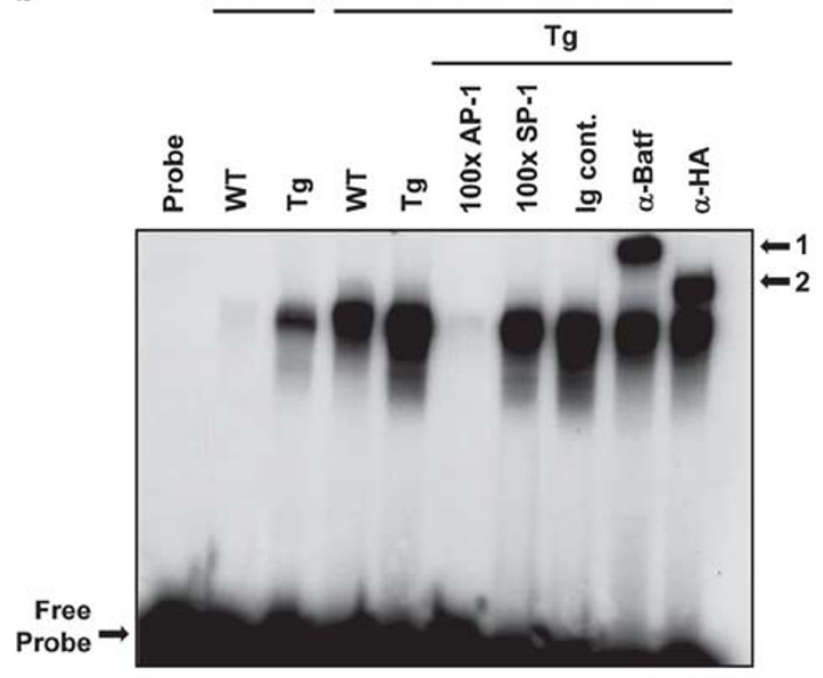

d
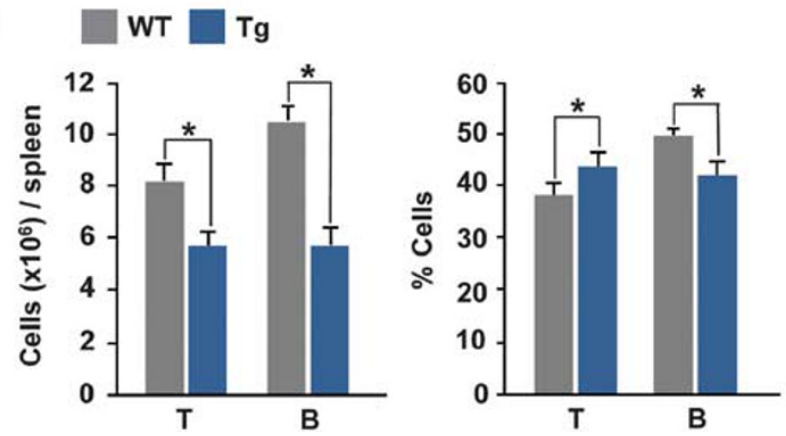
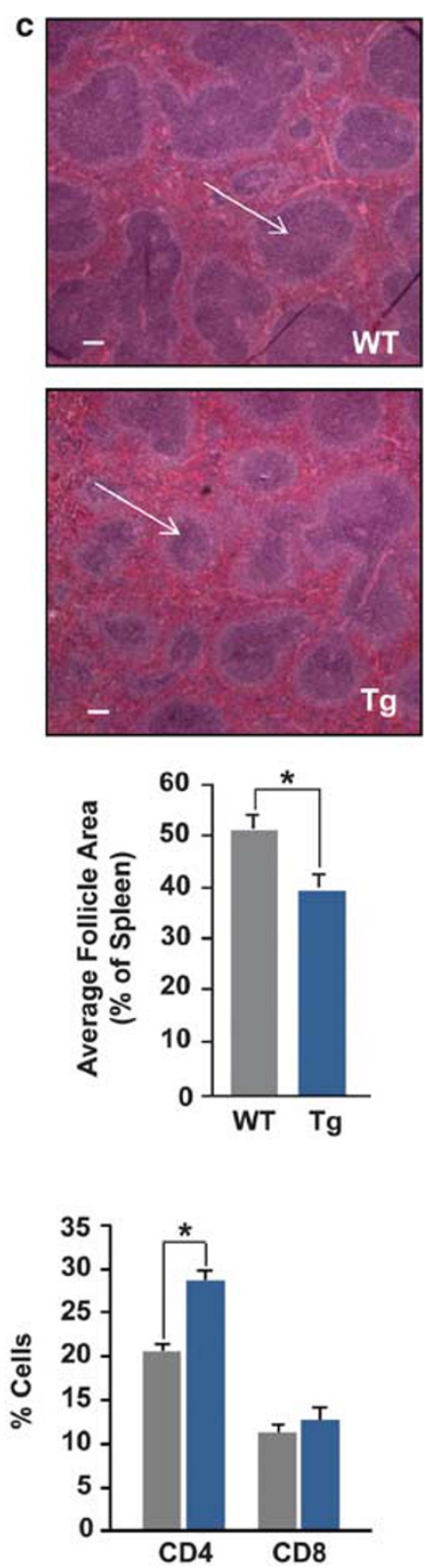

Figure 1 Characterization of the CD2-HA-BATF Tg mice. (a) Representative immunoblot of protein extracts prepared from activated splenic T cells from the indicated mice - WT, CD2-HA-BATF (Tg) and Batf ${ }^{\wedge Z / \triangle Z}$ (null) - probed with anti-Batf antiserum (top) or with anti- $\beta$-actin antibody as a control (bottom). (b) Representative EMSA using a radiolabelled AP-1 DNA probe and nuclear extracts from WT and CD2-HA-BATF (Tg) splenocytes stimulated in vitro with anti-CD3 and CD28 antibodies or with vehicle (no stim) as a control. Competition with 100-fold molar excess of unlabeled AP-1, but not SP-1, consensus DNA identifies the shifted complexes as AP-1. Addition of control $\mathrm{IgG}$ anitibody (Ig cont.), anti-Batf antiserum ( $\alpha$-Batf) or anti-HA antiserum $(\alpha-\mathrm{HA})$ confirms participation of Batf (endogenous and HA-tagged) in the complexes. Migration of these antibody supershifted complexes varies with the antibody used and are indicated by the arrows (1 and 2$)$ on the right. (c) Representative photomicrographs $(\times 4)$ of $\mathrm{H} \& \mathrm{E}$ stained spleen sections reveal a smaller white pulp area (arrows) in CD2-HA-BATF (Tg) versus WT mice (bars $=100 \mu \mathrm{m})$. Cumulative data from sections in which white pulp area was measured and expressed as a percent of the total spleen area are graphed below. $\left(N=4 ;{ }^{*} P<0.025\right)$. (d) Flow cytometry was used to quantify $B$ and $T$ cells in splenocytes prepared from CD2-HA-BATF (Tg) and WT mice. Left panel employs anti-TCR $\beta$ and anti-B220 antibodies to quantify total T cells and B cells per spleen, respectively $\left(N=8 ;{ }^{*} P<0.0008\right)$. Center panel expresses these same data as a percent of total splenoctyes $\left(N=8 ;{ }^{*} P<0.05\right)$. Right panel employs anti-CD4 and anti- CD8 antibodies to establish the percent of single-positive T cells $\left(N=8 ;{ }^{*} P<5 \times 10^{-7}\right)$

from 15 individual tumors was analyzed and all samples exhibited a polyclonal banding pattern similar to that of tumors 1, 2 and 3 presented in Figure $3 \mathrm{~b}$. These results confirm the polyclonal origin of the $T$ cells that populate these lesions.

To investigate the mechanism underlying tumor formation in CD2-HA-BATF Tg mice, single-cell suspensions of tumor tissue were cultured in the presence of bromodeoxyuridine (BrdU) and DNA replication quantified by enzyme-linked immunosorbent assay (ELISA). Cells from the lymph nodes of WT littermates were used as controls. Previous studies had demonstrated that BATF expression inhibits the proliferation of $T$ cells in vitro ${ }^{5,14}$ and the results shown in Figure $3 c$ confirm 
that Tg-tumor cells do not proliferate as well as WT controls. These data provide convincing evidence that the LPD detected in aged CD2-HA-BATF mice cannot be explained by an increase in the proliferative capacity of the BATFexpressing cells.

If the accumulation of $T$ cells in these mice cannot be attributed to increased proliferation, perhaps it is due to an impaired apoptotic response that, over time, allows T cells that would normally be eliminated during the course of an adaptive immune response to persist and accumulate in lymphoid tissue. To address this hypothesis, purified CD4 ${ }^{+} \mathrm{T}$ cells from the spleens of 8-12-week-old CD2-HA-BATF mice were exposed to an apoptotic stimulus in vitro and the death response of the culture was measured by the immunodetection of cleaved Pro-caspase 3. Initially, the response of the cells to extrinsic apoptotic signals was examined and the
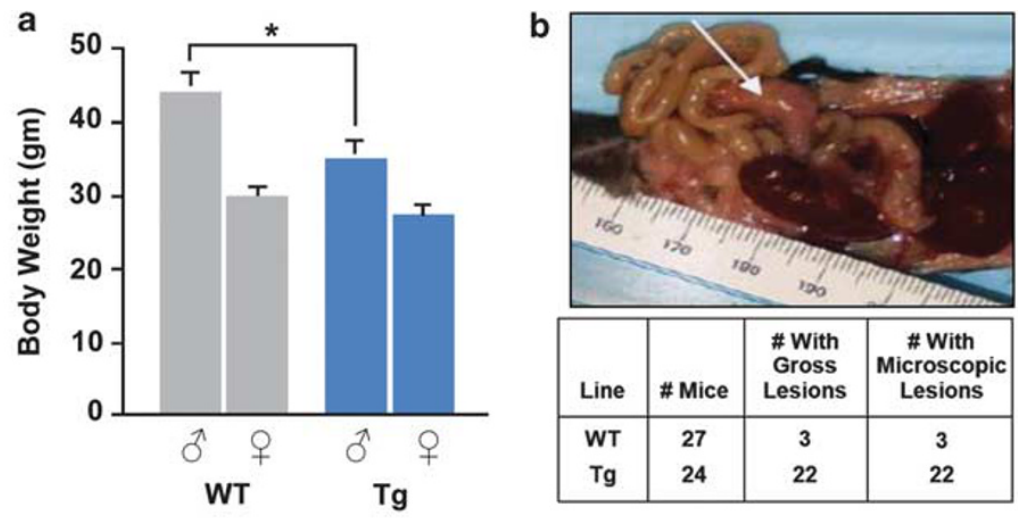

c
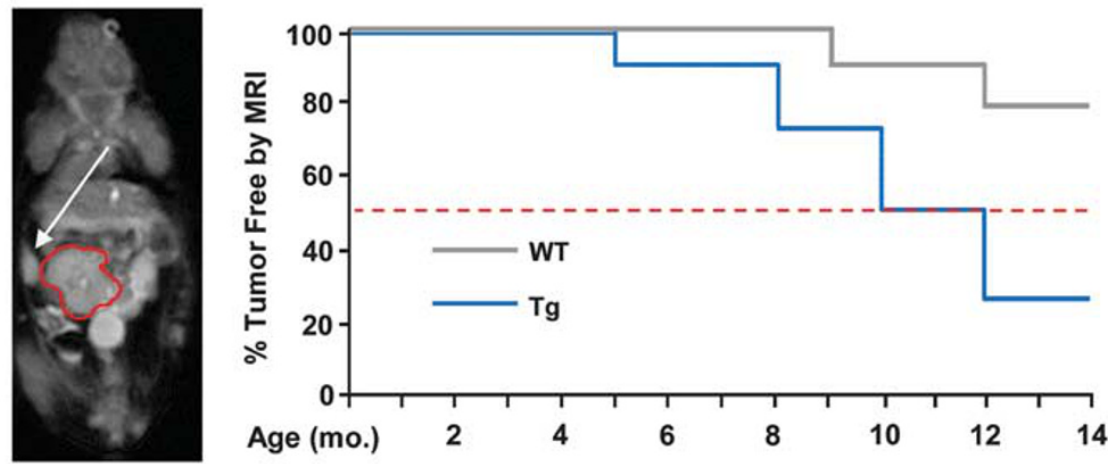

d


e

results demonstrated that activation-induced cell death was equivalent for $\mathrm{Tg}$ and WT $\mathrm{T}$ cells (data not shown). Subsequently, an established in vitro protocol of cytokine withdrawal $^{16}$ was used to compare the activated cell autonomous death (ACAD) ${ }^{17}$ response of WT versus $\mathrm{Tg} \mathrm{T}$ cells. In this case, under conditions of IL-2 withdrawal, the presence of Caspase 3 was detected readily in control WT cells within $24 \mathrm{~h}$. In contrast, there was dramatically less Caspase 3 detected in lysates from Tg T cells, despite the fact that these cells appear to express more Pro-caspase 3 than their WT counterparts (Figure $3 d$ ). These data suggest that overexpression of BATF impacts the T-cell gene expression program in a manner that alters how $\mathrm{T}$ cells respond to intrinsic apoptotic signals.

Further support for apoptotic suppression by BATF was obtained using the terminal deoxynucleotidyl transferase dUTP nick end-labeling (TUNEL) assay. CD4 ${ }^{+}$cells were isolated and apoptosis in control and in IL-2 depleted cultures was quantified after $24 \mathrm{~h}$ by counting cells that stained positive for DNA strand breaks (Figure 4a). Assays were performed using WT cells, CD2-HA-BATF cells and cells isolated from Batf null mice. The cumulative data are presented in Figure 4b. Using the level of apoptosis measured for Tg cells following cytokine withdrawal as the baseline, WT cells expressing a normal level of Batf show a 2-fold increase in apoptosis and Batf null cells show a 4-fold increase. These data suggest a putative function for Batf as a molecular rheostat that determines the signal strength of apoptotic induction during ACAD.

The apoptotic response of $\mathrm{T}$ cells to trophic factor withdrawal relies on the differential expression of Bcl-2 family proteins. ${ }^{17-19}$ Notably, to coordinate the mitochondrial events necessary to trigger Cytochrome $c$ release and the subsequent activation of Caspases, transcriptional and posttranslational mechanisms are used to decrease the ratio of the anti-apoptotic protein, Bcl-2, to the pro-apoptotic protein, Bim. $^{20}$ To evaluate the impact of Batf on Bcl-2 and Bim transcripts, WT, Tg and Batf null CD4 ${ }^{+}$T cells were isolated and gene expression changes captured in RNA prepared before, and $12 \mathrm{~h}$ following, IL-2 withdrawal. Semi-quantitative PCR and RT-qPCR were performed to detect and to quantify $\mathrm{Bcl}-2$ and BimEL MRNA, respectively (Figure 4c). The $\mathrm{RT}$-qPCR results (Figure 4c, right panel) are graphed relative to transcript levels in WT cells before IL-2 withdrawal which were set to 1 (data not shown). Following IL-2 withdrawal, the
Tg cells do not fully downregulate Bcl-2 mRNA and, accordingly, retain a low level of BimEL mRNA. Conversely, while there is no statistical difference in the level of $\mathrm{Bcl}-2$ mRNA in Batf null and WT cells, BimEL mRNA is 2-fold higher in Batf null cells than in WT cells. These observations favor a model in which Batf participates in nuclear events that promote an anti-apoptotic gene expression program and suppress the intrinsic, T-cell apoptotic response.

\section{Discussion}

This study describes a novel function for the AP-1 transcription factor, Batf, as a mediator of nuclear events contributing to the suppression of ACAD in $\mathrm{CD}^{+}{ }^{+} \mathrm{T}$ cells. We propose that, over time, the resistance to apoptotic signaling conferred on cells overexpressing Batf results in the accumulation of $\mathrm{T}$ cells in lymphoid tissues of CD2-HA-BATF $\mathrm{Tg}$ mice. The observation that the tumors arising in these mice are polyclonal is consistent with this model and defines the condition as T-cell LPD as opposed to T-cell lymphoma. The etiology of the vast majority of human LPDs remains unknown. ${ }^{21}$ One exception is autoimmune lymphoproliferative syndrome (ALPS), ${ }^{22}$ which is characterized by a defect in the extrinsic (i.e., death receptor mediated) apoptotic pathway. BATF drives formation of an LPD in mice by suppressing the T-cell response to intrinsic apoptotic signaling. This finding suggests an additional molecular mechanism that should be investigated in the ongoing effort to further classify human LPD.

As the name implies, ALPS presents as a polyclonal accumulation of $T$ cells and is associated with high levels of autoantibody production. ${ }^{22}$ In this regard, previous work with Batf null mice revealed an immunoglobulin-deficient phenotype produced as a consequence of combined defects in Th17 and Tfh-cell development and in the mechanism of Ig classswitch recombination. ${ }^{10}$ It has been demonstrated that Batf null mice are resistant to experimental autoimmune encephalomyelitis $(\mathrm{EAE})^{7}$ and therefore, one might predict that $\mathrm{EAE}$ would be accelerated in mice that overexpress Batf. Although we have not examined this response in CD2-HA-BATF mice, we have compared the resting levels of immunoglobulin in sera from $\mathrm{Tg}$ mice and nontransgenic (WT) littermates. Results show that all of the class-switched Ig subclasses examined in these mice are increased $2-4$ fold (Figure $5 a$ ). To investigate if self-reactive antibodies comprise a significant

Figure 2 Gross characterization of CD2-HA-BATF Tg tumors. (a) WT and CD2-HA-BATF (Tg) mice were aged 10 to 12 months. Body weights in grams (g) were determined at time of killing and the average weights plotted. Male Tg mice display a lower average body weight compared with $W T$ males $\left(N=6 ;{ }^{*} P<0.03\right)$, whereas the very modest decrease in the weight of female $\operatorname{Tg}(N=9)$ versus WT $(N=8)$ mice is not significant $(P=0.10)$. Bars represent $\mathrm{S}$.E. (b) Mice of the indicated genotype, aged 10 to 12 months, were examined at necropsy for gross lesions. A typical tumor (arrow) is shown with a mm ruler for size reference. Twenty-two of 24 ( $>91 \%$ ) CD2-HA-BATF $(\mathrm{Tg})$ mice demonstrated gross lesions together with peripheral lymphadenopathy in at least one node and microscopic lesions in liver and pancreas. Tumors were detected in only three WT control mice (11\%). (c) Eleven CD2-HA-BATF $(\mathrm{Tg})$ and 10 WT mice were subject to MRI at monthly intervals starting at 4 months to monitor tumor formation. A representative, T2 weighted MRI of a 10-month Tg mouse (left panel) highlights a discrete mesenteric lymph node tumor (circled in red) and the presence of splenomegaly (arrow). The kinetics of tumor detection in Tg and WT animals (right panel) indicate that the median age of tumor onset in Tg mice (dotted line) is 10 months. (d) Representative photos of H\&E stained sections contrast the normal architecture of a lymph node from a 10-month-old WT mouse (upper left) with the architecture of a lymphoid tumor identified in an aged Tg mouse (upper right). Serial sections of the same Tg tumor (visualized using $\mathrm{H}$ only) were processed for IHC and stained with anti-CD3 to detect T cells, anti-CD79a to detect B cells and anti-HA to detect the expression of the HA-BATF protein. The bottom right panel is the inset of the anti-HA IHC staining on the bottom left (box). Images are $\times 10$ magnification (bar $=40 \mu \mathrm{m}$ ) except for the inset image which is $\times 40$ (bar $=10 \mu \mathrm{m}$ ). (e) Flow cytometry was used to quantify the percent Tg-tumor $\mathrm{T}$ cells expressing HA-BATF. Cells were stained with anti-CD3 $\varepsilon$ and anti-HA antibodies. A representative histogram from cells gated on $\mathrm{CD} 3 \varepsilon{ }^{+}$cells shows that cells from the tumor (black) are HA-positive relative to control cells (red). The average percent of HA-positive cells per tumor $(N=3)$ is indicated 
a
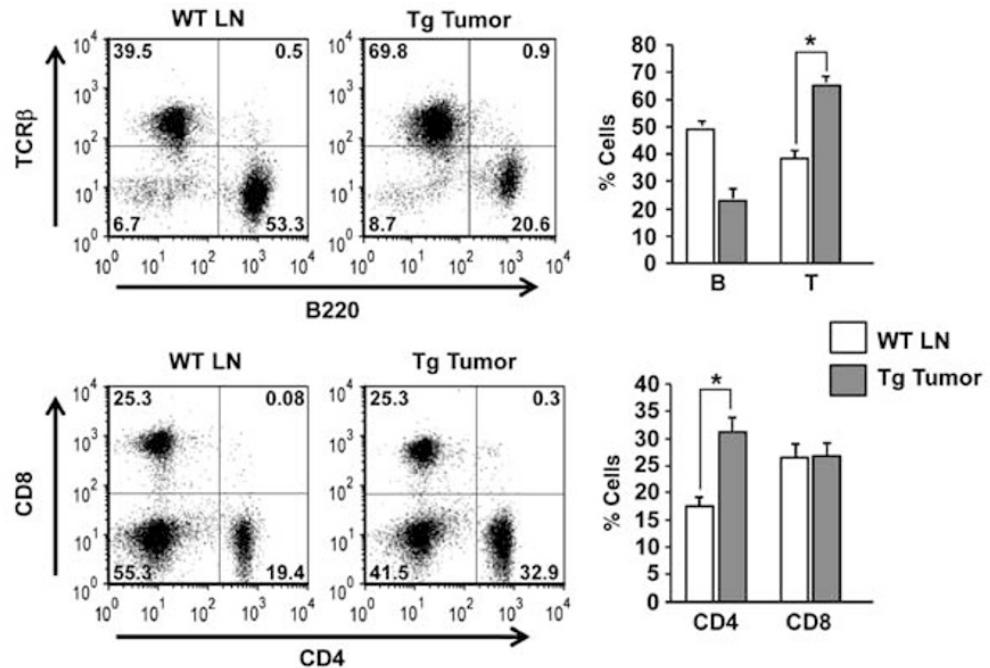

b
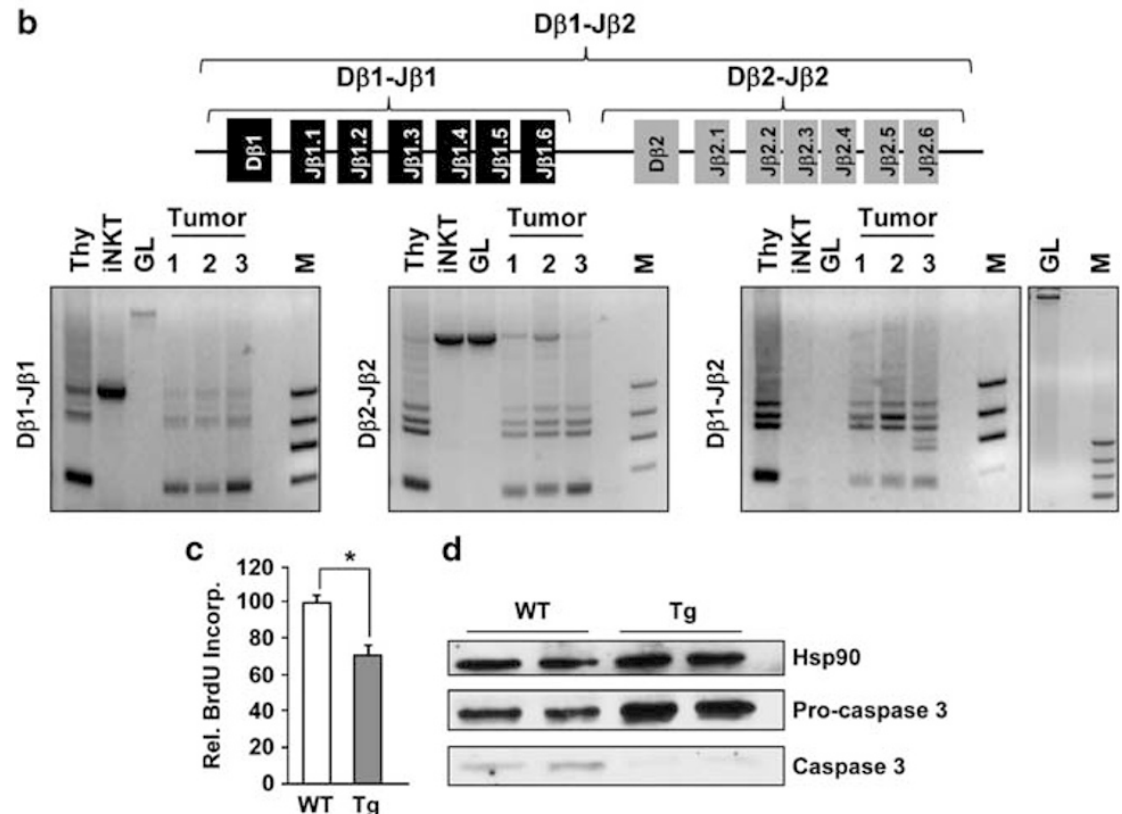

d

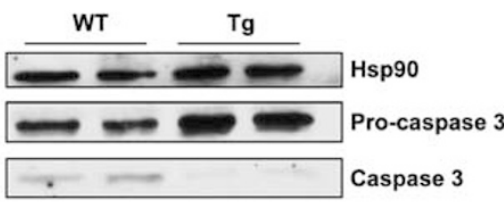

Figure 3 CD2-HA-BATF Tg-tumor T cells proliferate poorly and show reduced Pro-caspase 3 cleavage upon induction of ACAD. (a) Flow cytometry was used to analyze cell suspensions from CD2-HA-BATF Tg-tumor tissue surface stained for $\mathrm{B} 220, \mathrm{CD} 3 \varepsilon$, $\mathrm{CD} 4$, and $\mathrm{CD} 8$. Quadrant numbers from the flow plots and $y$ axis values from accompanying graphs indicate the percentage of $B$ versus T cells. CD2-HA-BATFTg tumors $(N=4)$ are composed primarily of $T$ cells $\left({ }^{*} P<5 \times 10^{-5}\right)$ with a bias towards the $\mathrm{CD} 4{ }^{+}$population $\left({ }^{\star} P<0.0004\right)$. Lymph node cell suspensions from age-matched, WT mice $(N=6)$ were used as controls. Bars denote S.E. (b) To assess tumor T-cell clonality, PCR was used to amplify three separate regions of the TCR $\beta$ locus D1-J2 domain (upper diagram) from DNA isolated from Tg tumors (Tumor 1,2,3). Results (lower panels) show a banding profile characteristic of a polyclonal T-cell population. Thymus DNA (Thy) is a control for all possible TCR $\beta$ D-J rearrangements and the iNKT cell line expresses a single TCR $\beta$ chain. ES $129 \mathrm{DNA}$ (GL) demonstrates the germ line, nonrearranged amplicon which for $\mathrm{D} \beta 1-\mathrm{J} \beta 2$ is resolved on a separate gel due to size. $\mathrm{M}=$ mol. wt. markers of $1,0.7,0.5$ and $0.3 \mathrm{kbp}$. Fifteen independent tumor samples were analyzed with comparable results. (c) $\mathrm{T}$ cells within single-cell suspensions prepared from $\mathrm{Tg}$ tumors or from the pooled peripheral lymph nodes of age-matched, WT mice were stimulated with anti-CD3 and anti-CD28 and evaluated for proliferation by measuring BrdU uptake $\left(N=4,{ }^{*} P<0.005\right)$. Bars indicate S.E. (d) Anti-Caspase 3 immunoblot of lysates prepared from WT and Tg CD4 ${ }^{+} \mathrm{T}$ cells following $24 \mathrm{~h}$ of IL-2 withdrawal to induce ACAD. Immunodetection of Hsp90 was used as the control. Lysates from two mice per genotype are shown. The experiment was performed four times with similar results

portion of this serum Ig, an ELISA for anti-nuclear antigen was performed. After normalizing for differences in total Ig content, more than a 2 -fold increase in reactivity was detected in the sera of CD2-HA-BATF Tg mice (Figure 5b). These data correlate nicely with our observations in Batf $f^{\Lambda Z / \Delta Z}$ mice ${ }^{10}$ and suggest that the Batf-induced alterations in T-cell homeostasis that lead to LPD also have a permissive effect on the development of autoimmune disease. Subjecting CD2-HA-
BATF Tg mice to experimental models of immune-mediated disease will be used as a further test of this possibility.

The findings from the present study and other descriptions of elevated Batf expression in human and mouse peripheral lymphoid tumors ${ }^{12,13}$ suggest that the influence of Batf on $\mathrm{AP}-1$ activity and on the transcription of specific genes has a role. ACAD, the apoptotic process that is impaired in CD2-HA$B A T F T$ cells, is associated with both transcriptional and post- 
a

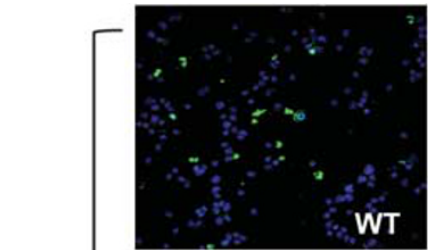

- IL-2
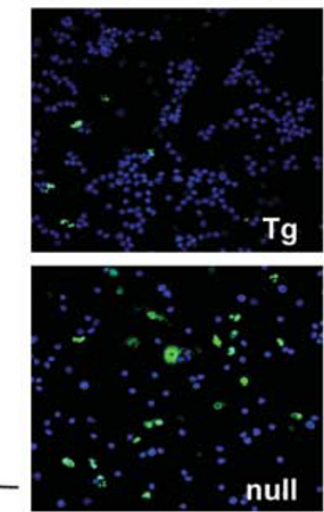

C



b

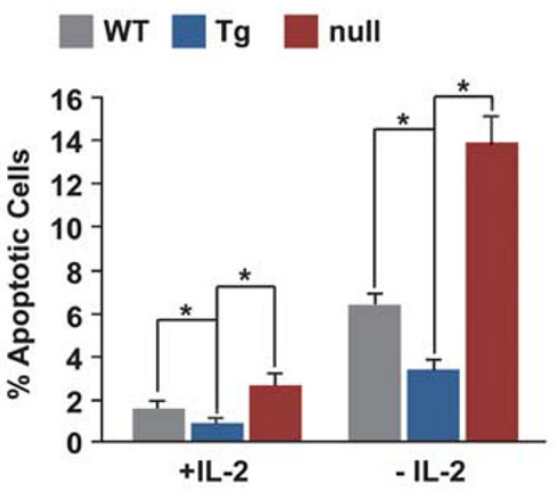

WT

$\mathrm{Tg}$

null

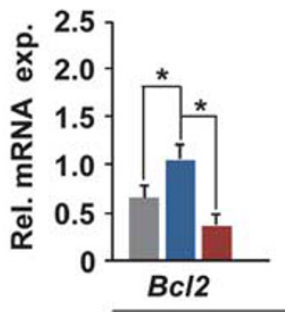

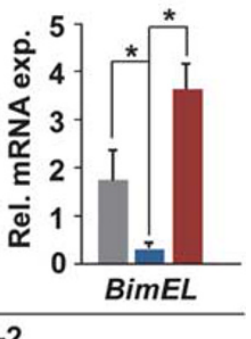

Figure 4 Impaired intrinsic apoptotic signaling in CD2-HA-BATFT cells. (a) CD4 ${ }^{+}$T cells from WT, CD2-HA-BATF (Tg), and Batf $4 / \Delta Z$ (null) mice were subjected to IL-2

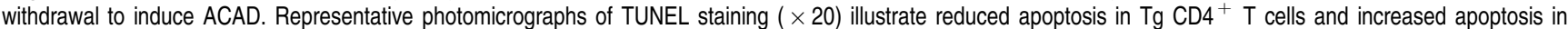
Batf ${ }^{\lrcorner Z / \triangle Z} \mathrm{CD}^{+}{ }^{+} \mathrm{T}$ cells, respectively. (b) The average \% apoptotic cells for each genotype ( $N=11, \mathrm{WT} ; N=6, \mathrm{Tg} ; N=6$, null) was calculated using the averages from three fields (as shown in (a)) per slide per mouse ( $\left.{ }^{\star} P<0.02\right)$. Bars indicate S.E. (c) Key transcripts involved in ACAD were detected and quantified using RNA isolated from the $\mathrm{CD} 4{ }^{+}$T cells of WT $(N=6), \mathrm{Tg}(N=6)$ and null $(N=6)$ mice before $(+)$ and after $(-)$ IL-2 withdrawal. The left panel is a representative gel showing the results from the semi-quantitative PCR analysis of Bcl-2 and BimEL transcript levels in the RNA of one mouse per genotype. Detection of $\beta$-actin transcripts is used as a control. The panels on the right contain the cumulative results of RT-qPCR analysis of all mouse samples (six per genotype) and reveal the impact of different levels of Batf expression on Bcl-2 and BimEL transcripts. For these experiments, detection of $H r p t m R N A$ was used as a control $\left({ }^{*} P<0.02\right)$. Bars indicate S.E.

translational events that increase the ratio of pro-apoptotic (e.g., Bim) to anti-apoptotic (e.g., Bcl-2) proteins and, by doing so, promote Cytochrome $c$ release by mitochondria and the activation of cytosolic pro-caspases. ${ }^{17,18,23,24}$ The shift from an anti- to a pro-apoptotic program in T cells occurs by a twostep process. The first step triggers Bcl-2 levels to decline as result of changes in the cytokine environment ${ }^{25}$ or increases in reactive oxygen species (ROS). ${ }^{26,27} \mathrm{Bcl}-2$ downregulation is not sufficient, however, and apoptosis requires a second step that is mediated by an increase in Bim expression. As prior studies demonstrate that BATF functions as an AP-1 inhibitor, ${ }^{4,5}$ the influence of BATF overexpression on decreasing $\mathrm{Bcl}-2$ levels is probably indirect. On the other hand, the reduced level of the BimEL transcript in CD2-HA-BATF Tg $T$ cells and the dramatically elevated level of BimEL mRNA in Batf null cells are both consistent with a direct role for Batf as a negative regulator of Bim transcription. Interestingly, apoptosis following trophic factor withdrawal in neurons has been shown to rely on the induction of Bim transcription by a c-Jun containing AP-1 complex that binds directly to an AP-1 motif in the Bim promoter. ${ }^{28,29}$ Furthermore, in hepatocytes, the generation of ROS triggers apoptosis by promoting the Erkdependent induction of C-Fos and increased Bim transcription through this same AP-1 site. ${ }^{30}$ Batf is not expressed neurons or hepatocytes making it worthwhile to identify the components of the AP-1 complex that are responsible for upregulating BimEL mRNA in T cells and establishing how Batf may alter the activity of this AP-1 complex to inhibit the induction of Bim gene transcription during ACAD.

Our analysis of the Batf-associated apoptotic defect has focused on $\mathrm{CD}^{+}{ }^{+} \mathrm{T}$ cells largely because of the CD4 ${ }^{+} \mathrm{T}$-cell bias displayed by the tumors. However, this does not rule out that apoptotic pathways in peripheral $C D 8^{+} \mathrm{T}$ cells from $C D 2$ $H A-B A T F$ and Batf null mice also are affected. In fact, while this work was in its final stages, Kuroda et al. ${ }^{11}$ published a study in which WT and Batf null mice were used to establish a 
a

\section{$\square$ WT \\ $\mathrm{Tg}$}



b
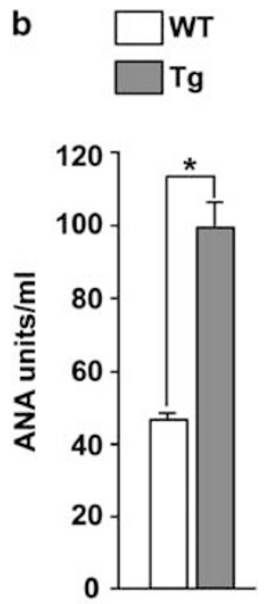

Figure 5 Hypergammaglobulinemia in CD2-HA-BATF Tg mice is coupled with indicators of autoimmune disease. (a) ELISA was performed on sera isolated from WT and CD2-HA-BATF (Tg) mice to measure the relative levels of the indicated Ig isotypes. The results from five mice per genotype were averaged for IgM, IgG1, $\lg$ G2C and IgE; three mice per genotype were used for the averaged results for IgA $\left.{ }^{*} P<0.05\right)$. Bars indicate S.E. (b) ELISA was performed on sera isolated from WT and $\mathrm{Tg}$ mice to assess levels of nuclear antigen-reactive antibodies. The average $(N=3)$ is presented. $\left({ }^{\star} P<0.008\right)$. Bars indicate S.E.

role for Batf in the later stages of $\mathrm{CD}^{+}$effector T-cell differentiation and survival. In that study, Batf was shown to have a positive impact on cellular energy metabolism in $\mathrm{CD} 8^{+}$ $\mathrm{T}$ cells, largely by inhibiting expression of the histone deacetylase Sirt1 which, in turn, allowed for the expression of genes necessary for cell survival. At which stage the expression of Batf might affect Sirt1 and the proper epigenetic remodeling of loci critical to the function of $\mathrm{CD}^{+}{ }^{+} \mathrm{T}$-cell subsets is an interesting question. Also of interest are studies to establish if the tumor cells in CD2-HA-BATFTg mice reflect a T-memory phenotype, which might be predicted based on our results and those of Kuroda et al. ${ }^{11}$

The mouse model described here provides novel opportunities to investigate how mis-regulation of the AP-1 transcription factor in T cells contributes to the improper control of apoptotic pathways and to the development of LPD. The high penetrance of this BATF-driven condition, together with the ability to detect the onset of these tumors using noninvasive MRI, should allow for testing both genetic strategies and targeted therapeutics for their effectiveness against T-cell LPD.

\section{Materials and Methods}

Mice. C57BL/6 CD2-HA-BATF and Batf ${ }^{1 Z / \Delta Z}$ mice have been described previously ${ }^{10,14}$ and were maintained in a specific-pathogen free animal facility according to institutional guidelines. CD2-HA-BATF mice overexpress the human BATF protein fused at the amino terminus to HA. The human and mouse BATF proteins differ by five amino acids and all are conservative changes located outside the core basic leucine zipper domain. Mice used in experiments were between 8 and 12 weeks of age unless noted otherwise.

Histology and IHC. Tissues were processed for hematoxylin and eosin (H\&E) staining and IHC as described. ${ }^{31}$ Sections were viewed using an Olympus BX41 microscope (Olympus Corp., Center Valley, PA, USA) and photographed using a Microimager II camera (Qimaging, Surrey, BC, Canada) and Northern Eclipse software (EMPIX Imaging Inc., Mississauga, ON, Canada). The comparison of spleen white pulp was made at $\times 4$ magnification using Image $\mathrm{J}$ software (NIH, Bethesda, MD, USA) to circumscribe the areas of white pulp area within the total area of spleen. White pulp area was expressed as a percentage of total spleen area and was averaged from the analysis of four, independent preparations per genotype. IHC was performed using the following primary antibodies: anti-CD79a (clone\# HM57, Dako, Carpinteria, CA, USA), rabbit antihuman CD3 (Dako) and rat anti-HA (clone\# 3F10, Roche, Indianapolis, IN, USA) and signal-detection reagents from Vector Laboratories (Burlingame, CA, USA). IHC images are $\times 10$ magnification, except for the inset which is $\times 40$. This IHC panel was repeated for four different tumors with similar results.

Flow cytometry. Flow cytometry was performed using a FC500 cytometer (Beckman Coulter, Brea, CA, USA) and the following antibodies from BD Biosciences (San Diego, CA, USA): anti-TCR $\beta$ (clone\# H57-S97), anti-CD3 $\varepsilon$ (clone\# 145-2C11), anti-B220 (clone\# RA3-6B2), anti-CD4 (clone\# L3T4) and anti-CD8 (clone\# 53-6.7). The anti-HA antibody (clone\# 3F10) is from Roche. Data were analyzed using FCSExpress3 software (DeNovo Software, Los Angeles, CA, USA).

Clonality assay. Genomic DNA was isolated from 15 independent $C D 2-H A-$ BATF tumors, the thymus tissue of a C57BL/6 mouse, ES 129 cells and 431A11 iNKT cells ${ }^{32}$ using phenol/chloroform extraction and the Wizard DNA Clean-UP System (Promega, Madison, WI, USA). The DNA samples were analyzed for D-J rearrangements in the TCR $\beta$ locus using the primers and amplification conditions described by Kawamoto et al. ${ }^{15}$

Cell proliferation assay. DNA synthesis was measured using a BrdU Cell Proliferation Assay Kit (Millipore, Billerica, MA, USA). Single-cell T-cell suspensions were prepared from CD2-HA-BATF lymph node tumors and agematched, WT lymph nodes using $\mathrm{CD} 19^{+}$B-cell depletion (Miltenyi Biotech, Auburn, CA, USA). Cells were plated at $2 \times 10^{4}$ cells/well in 96 -well plates and stimulated with anti-CD3 and anti-CD28 antibodies as described. ${ }^{8}$ After $48 \mathrm{~h}, \mathrm{BrdU}$ was added for $24 \mathrm{~h}$ and labeled DNA quantified by ELISA.

Cytokine withdrawal experiments. Splenocytes were prepared and $\mathrm{CD}^{+}{ }^{+} \mathrm{T}$ cells isolated using a $\mathrm{CD} 4^{+} \mathrm{T}$-cell isolation kit (Miltenyi Biotec, Auburn, CA, USA). T-cell activation and cytokine withdrawal were performed as described. ${ }^{16}$ Briefly, T cells were activated for $72 \mathrm{~h}$ in $100 \mathrm{U} / \mathrm{ml} \mathrm{IL-2} \mathrm{(R \& D}$ Systems, Minneapolis, MN, USA), $5 \mu \mathrm{g} / \mathrm{ml}$ soluble anti-CD28 and plate-bound anti-CD3 $\varepsilon$ (BD Biosciences). Following $24 \mathrm{~h}$ of culture in IL-2 alone, the IL-2 was withdrawn. Samples for immunoblot and cytospin preparations were collected $24 \mathrm{~h}$ post-withdrawal. RNA samples were harvested $12 \mathrm{~h}$ post-withdrawal.

Immunoblots. To detect Batf protein, $T$ cells were isolated from spleen, stimulated with anti-CD3 and anti-CD28 antibodies as described ${ }^{8}$ and immunoblotted as described ${ }^{33}$ using rabbit anti-BATF serum. ${ }^{34}$ Reactivity with anti- $\beta$-actin antibody was used as the positive control. For Pro-caspase 3 and Capase 3 , protein isolated from $T$ cells that had been subjected to cytokine withdrawal was immunoblotted using rabbit anti-Caspase 3 antibody (Cell Signaling Technologies, Danvers, MA, USA). Detection of Hsp90 using rabbit antiHsp 90 antibody (Santa Cruz Biotechnology, Santa Cruz, CA, USA) was used as the positive control.

MRI. A monthly, longitudinal MRI study was performed using 11 CD2-HA-BATF mice and 10 sex-matched, WT littermates beginning at 4 month of age and ending at 14 months of age. The animals were imaged while sedated with $70-90 \mathrm{mg} / \mathrm{kg}$ ketamine and $5-10 \mathrm{mg} / \mathrm{kg}$ xylazine administered intraperitoneally. Images were acquired using a 3.0 Tesla General Electric Signa HDx scanner and a transmit/ receive knee array coil (GE Healthcare, Piscataway, NJ, USA).

TUNEL assay. Splenocytes from 9 WT, 6 CD2-HA-BATF and 6 $B$ Batf ${ }^{\wedge / \Delta Z}$ mice were enriched for CD4 ${ }^{+} \mathrm{T}$ cells by Macs (Miltenyi Biotech) and subjected to cytokine withdrawal as described above. After $24 \mathrm{~h}$, cells were transferred to slides using a CytoSpin 4 Cytocentrifuge (Thermo Fisher Scientific, Waltham, MA, USA). TUNEL of apoptosis-induced DNA damage was assessed using the In Situ Cell Death Detection Kit (Roche). TUNEL-positive cells were expressed as a percent of total cells (DAPI positive) per field at $\times 20$ magnification. Three fields per slide were counted and averaged to obtain the percent apoptotic $T$ cells for each mouse spleen. 
Analysis of mRNA expression. Splenocytes were enriched for $\mathrm{CD}^{+} \mathrm{T}$ cells and subjected to cytokine withdrawal as described above. After $12 \mathrm{~h}$, RNA was isolated and converted to CDNA. SYBR green (Roche) RT-qPCR was performed and results analyzed as described. ${ }^{10} \mathrm{Hprt}$ and $\beta$-actin primers have been described previously ${ }^{10}$ and primers for $B C l-2$ and BimEL are from Kuribara et al. ${ }^{35}$ Results were normalized to Hprt expression and $\Delta \Delta C$ t values used to calculate the relative expression level of each mRNA. Semi-quantitative RT-PCR was performed as described ${ }^{34}$ for 25 cycles using primers for $\beta$-actin, ${ }^{34}$ $B C l-2^{36}$ )and BimEL. ${ }^{35}$ DNA products were visualized by ethidium bromide staining following resolution by $3 \%$ agarose gel electrophoresis.

ELISA. Blood from mice was collected by cardiac puncture and allowed to clot at room temperature in Microtainer Serum Separator Tubes (BD Biosciences). Serum was isolated by centrifugation. ELISA for the $\lg M$, $\lg G 1, \lg G 2 C$, $\lg A$ and $\operatorname{lgE}$ isotypes were perfomed as previously described. ${ }^{10}$ Values were normalized and expressed as a fold-over the WT control, which was set to 1. ELISA for anti-nuclear antibodies (ANA) was performed using the Mouse ANA Ig ELISA Kit (Alpha Diagnostics, San Antonio, TX, USA). In this case, total protein content was normalized by adding $\mathrm{lg}$ to the non- $\mathrm{Tg}$ sera before assay. Values in units/ml were calculated from a standard curve using the reagents provided in the kit.

\section{Conflict of Interest}

The authors declare no conflict of interest.

Acknowledgements. The authors thank $\mathrm{J}$ Hallett for the generation and A Kaufmann, J Skiba, M Lawson and K Oates for the routine care of the mice used in the study. The authors also thank T Talavage, Director of the Purdue MRI facility, for help with the design and implementation of the imaging study. This work was supported by NIH grants CA782464 and CA114381 (EJT), the Indiana Elks Cancer Research Program (EJT), NIH T32 GM08298 (KLJ-W and MRL) and by a Career Development Award from the Indiana Clinical and Translational Sciences Institute (NIH 5TL1 RR025759) (MRL). Publication costs were partially covered by funds awarded to EJT by the Purdue University Center for Cancer Research.

1. Eferl R, Wagner EF. AP-1: a double-edged sword in tumorigenesis. Nat Rev Cancer 2003; 3: 859-868.

2. Hess J, Angel P, Schorpp-Kistner M. AP-1 subunits: quarrel and harmony among siblings. J Cell Sci 2004; 117(Part 25): 5965-5973.

3. Foletta VC, Segal DH, Cohen DR. Transcriptional regulation in the immune system: all roads lead to AP-1. J Leukoc Biol 1998; 63: 139-152.

4. Echlin DR, Tae HJ, Mitin N, Taparowsky EJ. B-ATF functions as a negative regulator of AP-1-mediated transcription and blocks cellular transformation by Ras and Fos. Oncogene 2000; 19: 1752-1763.

5. Williams KL, Nanda I, Lyons GE, Kuo CT, Schmid M, Leiden JM et al. Characterization of murine BATF: a negative regulator of activator protein-1 activity in the thymus. Eur J Immunol 2001; 31: 1620-1627.

6. Ise W, Kohyama M, Schraml BU, Zhang T, Schwer B, Basu U et al. The transcription factor BATF controls the global regulators of class-switch recombination in both $B$ cells and T cells. Nat Immunol 2011; 12: 536-543.

7. Schraml BU, Hildner K, Ise W, Lee WL, Smith WA, Solomon B et al. The AP-1 transcription factor Batf controls $\mathrm{T}(\mathrm{H}) 17$ differentiation. Nature 2009; 460: 405-409.

8. Williams KL, Zullo AJ, Kaplan MH, Brutkiewicz RR, Deppmann CD, Vinson C et al. BATF transgenic mice reveal a role for activator protein-1 in NKT cell development. J Immunol 2003; 170: 2417-2426.

9. Zullo AJ, Benlagha K, Bendelac A, Taparowsky EJ. Sensitivity of NK1.1-negative NKT cells to transgenic BATF defines a role for activator protein-1 in the expansion and maturation of immature NKT cells in the thymus. J Immunol 2007; 178: 58-66.

10. Betz BC, Jordan-Williams KL, Wang C, Kang SG, Liao J, Logan MR et al. Batf coordinates multiple aspects of $B$ and $T$ cell function required for normal antibody responses. J Exp Med 2010; 207: 933-942.

11. Kuroda S, Yamazaki M, Abe M, Sakimura K, Takayanagi H, Iwai Y. Basic leucine zipper transcription factor, ATF-like (BATF) regulates epigenetically and energetically effector CD8 T-cell differentiation via Sirt1 expression. Proc Natl Acad Sci USA 2011 108: $14885-14889$

12. Lorenzo Y, Provencio M, Lombardia L, Diaz R, Silva J, Herrera M et al. Differential genetic and functional markers of second neoplasias in Hodgkin's disease patients. Clin Cancer Res 2009; 15: 4823-4828.

13. Rasmussen MH, Sorensen AB, Morris DW, Dutra JC, Engelhard EK, Wang CL et al. Tumor model-specific proviral insertional mutagenesis of the Fos/Jdp2/Batf locus. Virology 2005; 337: 353-364.

14. Thornton TM, Zullo AJ, Williams KL, Taparowsky EJ. Direct manipulation of activator protein-1 controls thymocyte proliferation in vitro. Eur J Immunol 2006; 36: 160-169.

15. Kawamoto H, Ohmura K, Fujimoto S, Lu M, Ikawa T, Katsura $Y$. Extensive proliferation of $\mathrm{T}$ cell lineage-restricted progenitors in the thymus: an essential process for clonal expression of diverse T cell receptor beta chains. Eur J Immunol 2003; 33: 606-615.

16. Tanaka N, Kamanaka M, Enslen H, Dong C, Wysk M, Davis RJ et al. Differential involvement of p38 mitogen-activated protein kinase kinases MKK3 and MKK6 in T-cell apoptosis. EMBO Rep 2002; 3: 785-791.

17. Hildeman DA, Zhu Y, Mitchell TC, Kappler J, Marrack P. Molecular mechanisms of activated T cell death in vivo. Curr Opin Immunol 2002; 14: 354-359.

18. Arnold R, Brenner D, Becker M, Frey CR, Krammer PH. How T lymphocytes switch between life and death. Eur J Immunol 2006; 36: 1654-1658.

19. Bouillet P, O'Reilly LA. CD95, BIM and T cell homeostasis. Nat Rev Immunol 2009; 9: 514-519.

20. Ewings KE, Wiggins CM, Cook SJ. Bim and the pro-survival Bcl-2 proteins: opposites attract, ERK repels. Cell Cycle 2007; 6: 2236-2240.

21. Greiner T, Armitage JO, Gross TG. Atypical Lymphoproliferative Diseases. Hematol Am Soc Hematol Educ Program 2000 133-146.

22. Worth A, Thrasher AJ, Gaspar HB. Autoimmune lymphoproliferative syndrome: molecular basis of disease and clinical phenotype. Br J Haematol 2006; 133: 124-140.

23. Brenner D, Krammer PH, Arnold R. Concepts of activated T cell death. Crit Rev Oncol Hematol 2008: 66: 52-64.

24. Hildeman DA, Zhu Y, Mitchell TC, Bouillet P, Strasser A, Kappler J et al. Activated T cell death in vivo mediated by proapoptotic Bcl-2 family member Bim. Immunity 2002; 16: 759-767.

25. Khaled AR, Durum SK. Lymphocide: cytokines and the control of lymphoid homeostasis. Nat Rev Immunol 2002; 2: 817-830.

26. Purushothaman D, Sarin A. Cytokine-dependent regulation of NADPH oxidase activity and the consequences for activated T cell homeostasis. J Exp Med 2009; 206: 1515-1523.

27. Hildeman DA, Mitchell T, Kappler J, Marrack P. T cell apoptosis and reactive oxygen species. J Clin Invest 2003; 111: 575-581.

28. Biswas SC, Shi Y, Sproul A, Greene LA. Pro-apoptotic Bim induction in response to nerve growth factor deprivation requires simultaneous activation of three different death signaling pathways. J Biol Chem 2007; 282: 29368-29374.

29. Whitfield J, Neame SJ, Paquet L, Bernard O, Ham J. Dominant-negative c-Jun promotes neuronal survival by reducing BIM expression and inhibiting mitochondrial cytochrome $c$ release. Neuron 2001; 29: 629-643.

30. Ishihara $\mathrm{Y}$, Ito $\mathrm{F}$, Shimamoto $\mathrm{N}$. Increased expression of $\mathrm{c}-\mathrm{Fos}$ by extracellular signalregulated kinase activation under sustained oxidative stress elicits BimEL upregulation and hepatocyte apoptosis. FEBS J 2011; 278: 1873-1881.

31. Zhu L, Shi G, Schmidt CM, Hruban RH, Konieczny SF. Acinar cells contribute to the molecular heterogeneity of pancreatic intraepithelial neoplasia. Am J Pathol 2007; 171: 263-273.

32. Lantz $\mathrm{O}$, Bendelac $\mathrm{A}$. An invariant $\mathrm{T}$ cell receptor alpha chain is used by a unique subset of major histocompatibility complex class I-specific CD4 + and CD4-8- T cells in mice and humans. J Exp Med 1994; 180: 1097-1106.

33. Liao J, Humphrey SE, Poston S, Taparowsky EJ. Batf promotes growth arrest and terminal differentiation of mouse myeloid leukemia cells. Mol Cancer Res 2011; 9: 350-363.

34. Johansen LM, Deppmann CD, Erickson KD, Coffin WF 3rd, Thornton TM, Humphrey SE et al. EBNA2 and activated Notch induce expression of BATF. J Virol 2003; 77: 6029-6040.

35. Kuribara R, Honda H, Matsui $H$, Shinjyo $T$, Inukai $T$, Sugita $\mathrm{K}$ et al. Roles of Bim in apoptosis of normal and Bcr-Abl-expressing hematopoietic progenitors. Mol Cell Biol 2004; 24: 6172-6183.

36. Ohigashi T, Ueno M, Nonaka S, Nakanoma T, Furukawa Y, Deguchi N et al. Tyrosine kinase inhibitors reduce bcl-2 expression and induce apoptosis in androgen-dependent cells. Am J Physiol Cell Physiol 2000; 278: C66-C72.

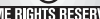
licensed under the Creative Commons Attribution-NonCommercial-No Derivative Works 3.0 Unported License. To view a copy of this license, visit http://creativecommons.org/licenses/by-nc-nd/3.0/ published by Nature Publishing Group. This work is Cell Death and Disease is an open-access journal 